\title{
miR-10a increases the cisplatin resistance of lung adenocarcinoma circulating tumor cells via targeting PIK3CA in the PI3K/Akt pathway
}

\author{
TONGHAI HUANG, KANGQI REN, GUANGGUI DING, LIN YANG, YUXIN WEN, \\ BIN PENG, GUANGSUO WANG and ZHENG WANG
}

Department of Thoracic Surgery, The Second Clinical Medical College (Shenzhen People's Hospital), Jinan University, Shenzhen, Guangdong 518020, P.R. China

Received March 19, 2019; Accepted December 13, 2019

DOI: $10.3892 / o r .2020 .7547$

\begin{abstract}
Circulating tumor cells (CTCs) that are shed from the primary tumor invade the blood stream or surrounding parenchyma to form new tumors. The present study aimed to explore the underlying mechanism of cisplatin resistance in lung adenocarcinoma CTCs and provide clinical treatment guidance for lung cancer treatment. CTCs from the blood samples of 6 lung adenocarcinoma patients were treated with different concentrations of cisplatin along with A549 and H1299 cells. The sensitivity of CTCs to cisplatin was explored by detecting the inhibitory rate via CCK- 8 assay. The related molecular mechanism was investigated by western blot analysis. miR-10a expression was detected using quantitative real-time PCR (RT-qPCR). The relationship between miR-10a and phosphatidylinositol-4,5-bisphosphate 3-kinase catalytic subunit $\alpha$ (PIK3CA) was verified and further confirmed by luciferase reporter assay, western blotting and RT-qPCR assay. The results revealed that CTCs exhibited lower cisplatin sensitivity than A549 and H1299 cells. Moreover, CTCs treated with cisplatin demonstrated higher miR-10a expression and lower PIK3CA expression than that in A549 and H1299 cells $(\mathrm{P}<0.01)$. Expression of phosphoinositide 3-kinase (PI3K) and protein kinase $\mathrm{B}(\mathrm{Akt})$ phosphorylation were also decreased in A549 and H1299 cells compared with CTCs after cisplatin treatment. PIK3CA is a target of miR-10a, and both miR-10a overexpression and PIK3CA knockdown obviously decreased the sensitivity of A549 and H1299 cells to cisplatin as well as the expression of PI3K and phosphorylation of Akt. PIK3CA overexpression attenuated the cisplatin resistance of A549
\end{abstract}

Correspondence to: Dr Tonghai Huang, Department of Thoracic Surgery, The Second Clinical Medical College (Shenzhen People's Hospital), Jinan University, 1017 East Gate Road, Shenzhen, Guangdong 518020, P.R. China

E-mail: tonghaihuang@aliyun.com

Key words: lung adenocarcinoma, circulating tumor cells, miR-10a, PIK3CA, cisplatin resistance and H1299 cells induced by miR-10a. In conclusion, miR-10a suppressed the PI3K/Akt pathway to strengthen the resistance of CTCs to cisplatin via targeting PIK3CA, providing a new therapeutic target for lung cancer treatment.

\section{Introduction}

Lung cancer, which originates from the bronchial mucosa or glands, is a malignant tumor with a high mortality rate. Approximately $27 \%$ of all tumor-related deaths are caused by lung cancer $(1,2)$. Non-small cell lung cancer (NSCLC) accounts for the majority (i.e., 85 to $90 \%$ ) of all lung cancer cases, where the incidence of lung adenocarcinoma has significantly increased to a greater extent than that of squamous cell carcinoma. In addition, it has become the most common type of lung cancer in many countries (3). With the development of medical technology, multiple chemotherapy drugs, such as platinum/pemetrexed (4), gefitinib (5), paclitaxel (6) and cisplatin (7), have been widely applied to treat lung cancer including lung adenocarcinoma. As an effective cytotoxic agent, cisplatin is a common first-line drug that is usually combined with other agents for the treatment of lung adenocarcinoma, but its efficacy is largely impaired by increasing chemoresistance (8). Therefore, it is necessary to further explore the underlying mechanisms of cisplatin chemoresistance in lung adenocarcinoma, which in turn facilitates the designing of treatment strategies for patients with lung adenocarcinoma.

Surgical resection is still the preferred treatment for patients with early-stage lung adenocarcinoma, but the 5-year survival rate of patients with stage I NSCLC is only $45-65 \%$ (9). Local recurrence occurs in approximately $6-11 \%$ of patients with stage I NSCLC, while distant metastasis accounts for approximately $23-30 \%$ of recurrence (10). Recurrence and metastasis are important factors in the poor prognosis of patients, and the presence of circulating tumor cells (CTCs) exacerbates these risks. CTCs were first reported by Thomas Ashworth (1869), and are defined as a set of cells that shed from the primary tumor and invade into the blood stream or surrounding parenchyma to form new tumors (11). Several studies have revealed that CTCs present dynamic changes in epithelial and mesenchymal phenotypes $(12,13)$ and an increase in the number of 
CTCs is usually correlated with tumor relapse and chemotherapy/radiotherapy resistance (14-16). At present, CTCs have been used as tumor biomarkers in various tumors including breast cancer, colorectal cancer and prostate cancer (17). However, the function and regulatory mechanisms related to CTCs in lung adenocarcinoma are rarely reported. MicroRNAs (miRNAs) are a class of small non-coding RNAs, with a length of 20-24 nt. Previous studies have demonstrated that several miRNAs, including miR-216a/miR-217 (18), miR-497 (19), the miR-200bc/429 cluster (20) and miR-31, are correlated with drug resistance and recurrence of cancer (18). In addition, miR-206 has been reported to regulate cisplatin resistance and epithelial-to-mesenchymal transition (EMT) in human lung adenocarcinoma cells partly through targeting MET (21). miR-15b was found to regulate cisplatin resistance and metastasis via targeting PEBP4 in human lung adenocarcinoma cells (22). Kitamura et al found that the miR-134/487b/655 cluster could regulate TGF- $\beta$-induced EMT and drug resistance to gefitinib by targeting MAGI2 in lung adenocarcinoma cells (23). miR-10a is a member of the highly conserved miR-10 family, and has been confirmed to be involved in drug resistance in several types of cancers. For example, miR-10a, miR-195, and miR-455-3p are correlated with temozolomide resistance in glioblastoma multiforme cells (24). Bao et al reported that serum miR-10a-5p and miR-196a-5p could serve as non-invasive biomarkers for NSCLC patients (25). Long non-coding RNA RP11-838N2.4 was found to increase the effect of temozolomide in glioblastoma cell lines via suppressing the function of miR-10a (26). Recently, Sun et al revealed that miR-10a knockdown could increase the cisplatin sensitivity of A549 cells via the TGF- $\beta / \mathrm{Smad} / \mathrm{STATA} 3$ signaling pathway (27). However, the function of miR-10a in the resistance of CTCs in lung adenocarcinoma to cisplatin has not been elucidated to date.

Therefore, in the present study, CTCs were isolated from the blood samples of lung adenocarcinoma patients to reveal the function of miR-10a in cisplatin resistance by when comparing with this function demonstrated in A549 and H1299 adenocarcinoma cells. In addition, the downstream target of miR-10a and the underlying signaling pathway involved in this progression were also determined. Based on these results, we hope to provide new information for understanding the cisplatin resistance of lung adenocarcinoma and new insights in the treatment of lung adenocarcinoma.

\section{Materials and methods}

Patients and sampling. Blood samples from 6 lung adenocarcinoma patients at the Shenzhen People's Hospital were collected from May to December 2017. The patients enrolled in this study underwent pathological diagnosis and radiography to confirm the presence of lung adenocarcinoma, and all underwent chemotherapy. All patients signed informed consent forms, and this study was authorized by the Ethics Committee of Shenzhen People's Hospital, The Second Clinical Medical College of Jinan University (Shenzhen, China).

Isolation of CTCs. Isolation of human CTCs was performed as previously reported by Gong et al (28). Briefly, 20-50 ml fresh blood was obtained from lung adenocarcinoma patients and layered over Ficoll-Paque at a density of $1.077 \mathrm{~g}$, and centrifuged at $400 \mathrm{x} \mathrm{g}$ for $30 \mathrm{~min}$ at room temperature. The cells in the interphase, including lymphocytes, monocytes, and tumor cells, were then resuspended in $300 \mu \mathrm{l}$ of solution (consisting of $100 \mu \mathrm{l}$ each of FcR blocking reagent, CD45 microbeads, and CD15 microbeads; purchased from Miltenyi Biotec; Germany) at a density of $5 \times 10^{7}$ cells. Following this, CD45 and CD15 microbeads were removed using the magnetic auto MACS ${ }^{\mathrm{TM}}$ Pro Separator (Miltenyi Biotec). Then, $100 \mu 1$ of CD326 (Miltenyi Biotec) per $5 \times 10^{7}$ cells was added and incubated at $4^{\circ} \mathrm{C}$ for $30 \mathrm{~min}$. Subsequently, the magnetic $\mathrm{CD} 326^{+}$and CD326- cells were eluted as epithelial cell adhesion molecule $(\mathrm{EpCAM})^{+}$and EpCAM- CTCs. Purification of epithelial cells was performed by immunofluorescent staining with anti-cytokeratin antibody (product code ab756; Abcam) to reach up to 95\% confluence. CTCs were identified when they presented positive staining by a tumor-specific marker cytokeratin and positive scoring based on the review of a cytopathologist.

Cell culture. Human lung adenocarcinoma cell lines A549 and H1299 were purchased from the Chinese Academy of Sciences Committee on Type Culture Collection Cell Bank (Shanghai, China) and maintained in Dulbecco's modified Eagle's medium (DMEM, Gibco; Thermo Fisher Scientific, Inc.) containing $10 \%$ fetal bovine serum (FBS, Gibco; Thermo Fisher Scientific, Inc.). CTCs were maintained in RPMI-1640 medium supplemented with $20 \mathrm{ng} / \mathrm{ml}$ basic FGF (Life Technology; Thermo Fisher Scientific, Inc.), $20 \mathrm{mg} / \mathrm{ml}$ EGF (Life Technology; Thermo Fisher Scientific, Inc.), $10 \mathrm{ml}$ B27 (Life Technology; Thermo Fisher Scientific, Inc.), and 1X antibiotic/antimycotic (Life Technology; Thermo Fisher Scientific, Inc.). All cells were maintained in a humidified incubator at $37^{\circ} \mathrm{C}$ with $5 \% \mathrm{CO}_{2}$ in atmosphere.

Cell transfection. A549 and H1299 cells were seeded in 6-well plates at a confluency of 40-60\% overnight. Then, $50 \mathrm{~nm}$ of miR-10a mimic (Guangzhou RiboBio Co., Ltd.) was transfected into A549 and H1299 cells using Lipofectamine 2000 (Invitrogen; Thermo Fisher Scientific, Inc.) according to the manufacturer's instructions. In addition, cells transfected with the negative control (NC) sequence served as the negative control. After that, the cells were harvested after transfection for $48 \mathrm{~h}$ and used for the following confirmation and investigation. For pcDNA3.1-PIK3CA, PIK3CA shRNA and its corresponding scramble sequences (Guangzhou RiboBio Co., Ltd.) were transfected into A549 and H1299 cells using Lipofectamine 2000 according to the manufacturer's instructions. After transfection for $48 \mathrm{~h}$, the cells were harvested for further validation and investigations.

CCK- 8 assay. The half maximal inhibitory concentration $\left(\mathrm{IC}_{50}\right)$ of each cell line to cisplatin was evaluated by CCK-8 assay. Briefly, the cells were seeded into 96 -well plates at a density of $5 \times 10^{3}$ cells per well for overnight. The cells were then treated with different concentrations of cisplatin $(0,0.05$, $0.1,0.5,1.0,5.0,10.0$ and $50.0 \mu \mathrm{g} / \mathrm{ml})$ for $24 \mathrm{~h}$. Following this, $10 \mu \mathrm{l}$ of CCK-8 solution (Boster) was added into each well and incubated for $4 \mathrm{~h}$ at $37^{\circ} \mathrm{C}$. Subsequently, the absorbance of each well was determined using a microplate reader (BioTek ELx800; BioTek Instruments, Inc.) at $450 \mathrm{~nm}$. Each sample 
Table I. General characteristics of the 6 patients with lung adenocarcinoma from whom CTCs were collected.

\begin{tabular}{lcccccc}
\hline & \multicolumn{5}{c}{ Patients } \\
\cline { 2 - 6 } Characteristics & $\# 1$ & $\# 2$ & $\# 3$ & $\# 4$ & $\# 5$ & \#6 \\
\hline Sex & $\mathrm{F}$ & $\mathrm{M}$ & $\mathrm{F}$ & $\mathrm{F}$ & $\mathrm{M}$ & $\mathrm{F}$ \\
Age (years) & 64 & 63 & 66 & 64 & 71 & 64 \\
Tumor size (mm) & 13 & 8 & 22 & 16 & 24 & N \\
Node status & $\mathrm{N} 1$ & $\mathrm{~N} 1$ & $\mathrm{~N} 2$ & $\mathrm{~N} 1$ & $\mathrm{M} 2$ & $\mathrm{~N} 1$ \\
Metastasis & $\mathrm{M} 0$ & $\mathrm{M} 0$ & $\mathrm{M} 1$ & $\mathrm{II}$ & $\mathrm{III}$ & M0 \\
Clinical stage & $\mathrm{II}$ & $\mathrm{II}$ & $\mathrm{IV}$ & 5 & 25 & 15 \\
CTC counts & 8 & 10 & 55 & $2017 / 9 / 15$ & $2017 / 11 / 1$ & $2017 / 11 / 21$ \\
Date of surgery & $2017 / 5 / 13$ & $2017 / 6 / 7$ & $2017 / 8 / 18$ & & & \\
\hline
\end{tabular}

F, female; M, male; CTCs, circulating tumor cells.

was designed with five repeats and each experiment was performed at least three times.

Luciferase activity assay. A549 cells were seeded in 6-well plates overnight and co-transfected with miR-10 $\mathrm{mimic} / \mathrm{NC}$ and psiCHECK2-PIK3CA-3'-UTR-WT (WT)/psiCHECK2-PIK3CA-3'-UTR-MUT (MUT) using Lipofectamine 2000 (Invitrogen) according to the manufacturer's protocol. After $24 \mathrm{~h}$ of transfection, luciferase activity was measured using dual-luciferase reporter assay (Promega) according to the manufacturer's instructions and Firefly luminescence was normalized to Renilla luminescence. Plasmids for this assay were purchased from Guangzhou RiboBio Co., Ltd.

Quantitative real-time PCR (RT-qPCR). After transfection or treatment, total RNA in the cells was isolated using TRIzol reagent (Takara). Then, RNA was reverse transcribed into cDNA using Bestar qPCR RT Kit (DBI, China) according to the manufacturer's protocol. Using cDNA as a template, amplification of genes was performed using DBI Bestar ${ }^{\circledR}$ Sybr Green qPCR Master Mix (DBI) according to the manufacturer's instructions on an Agilent Stratagene Mx2000P PCR instrument (Agilent Technologies) under the following conditions: $95^{\circ} \mathrm{C}$ for $2 \mathrm{~min}$ and 40 cycles of $94^{\circ} \mathrm{C}$ for $20 \mathrm{sec}, 58^{\circ} \mathrm{C}$ for $20 \mathrm{sec}$, and $72^{\circ} \mathrm{C}$ for $20 \mathrm{sec}$. Primers of genes were designed as follows: miR-10a, forward, 5'-ACACTCCAGCTGGGTACCCTGTAGATCCGAAT-3' and reverse, 5'-CTCAACTGGTGTCGTGGAGTCGGCAATTCA GTTGAGCACAAATTC-3'; U6, forward, 5'-CTCGCTTCG GCAGCACA-3' and reverse, 5'-AACGCTTCACGAATTTGC GT-3'. Subsequently, the relative gene expression was analyzed using the $2^{-\Delta \Delta \mathrm{Cq}}$ method by normalizing to U6 (29).

Western blotting. After transfection or treatment, the cells were lysed using RIPA lysis buffer (Pierce; Thermo Fisher Scientific, Inc.) supplemented with a Protease Inhibitor Cocktail (Pierce; Thermo Fisher Scientific, Inc.). Then, the protein concentration of the lysates was determined using the BCA method (Pierce; Thermo Fisher Scientific, Inc.). Following this, the protein solution was boiled with equiva- lent amounts of loading buffer for $10 \mathrm{~min}$, equal amounts of protein $(30 \mu \mathrm{g})$ were subjected to $8-12 \%$ SDS-PAGE gel electrophoresis, and then transferred onto a PVDF membrane (Millipore). Membranes were blocked with 5\% non-fat milk in Tris-buffered saline and incubated with anti-PIK3CA (product code ab40776; dilution 1:1,000), PI3K (product code ab32089; dilution 1:1,000), Akt (product code ab179463; dilution 1:1,000), phosphorylated (p)Akt (product code ab81283; dilution 1:5,000), or GAPDH (product code ab181602; dilution 1:5,000; Abcam) antibodies at $4^{\circ} \mathrm{C}$ overnight followed by horseradish peroxidase-conjugated secondary antibody (product code ab205718; dilution 1:10,000; Abcam). Subsequently, the protein bands in the membranes were visualized using the ECL method (Pierce; Thermo Fisher Scientific, Inc.) The gel optical processing system (Image-Pro Plus 6.0; Media Cybernetics) was used to analyze the net optical density of the bands.

Statistical analyses. All statistical analyses were performed using SPSS 16.0 (SPSS Inc.) and GraphPad Prism 6.0 (GraphPad Software). All experiments were performed in triplicate and the average values were calculated and are presented as mean \pm standard deviation. Comparisons between two groups were estimated using unpaired t-test or Mann-Whitney $\mathrm{U}$ test. $\mathrm{P}<0.05$ was assigned to indicate statistical significance.

\section{Results}

CTCs present a lower sensitivity to cisplatin than the A549 and H1299 cell lines. A total of 6 lung adenocarcinoma patients were enrolled in this study, including two males and four females average aged is 65.3 years. Their clinical stages ranged from stage II to stage IV. The clinical characteristics of the 6 adenocarcinoma patients are summarized in Table I. CTCs were isolated from 6 adenocarcinoma patients, and A549 and H1299 cells were treated with different concentrations of cisplatin to determine the proliferation of each cell line using CCK- 8 assay. The results presented that the $\mathrm{IC}_{50}$ values of the CTCs from 6 patients $\left(\mathrm{IC}_{50}=31.14,25.54,25.50,20.73,17.39\right.$, and 21.96, respectively) were significantly higher than that of 

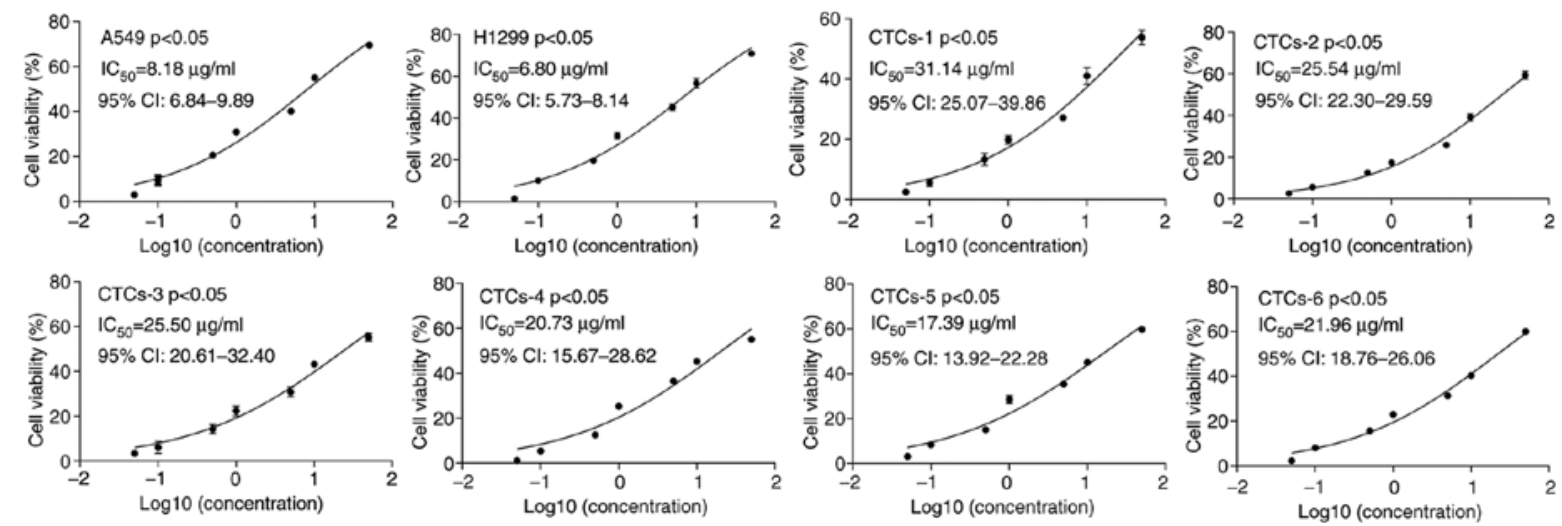

Figure 1. Inhibitory rates for cisplatin treatment in lung adenocarcinoma A549 and H1299 cells and CTCs from 6 patients. CTCs, circulating tumor cells; IC $_{50}$, half maximal inhibitory concentration.
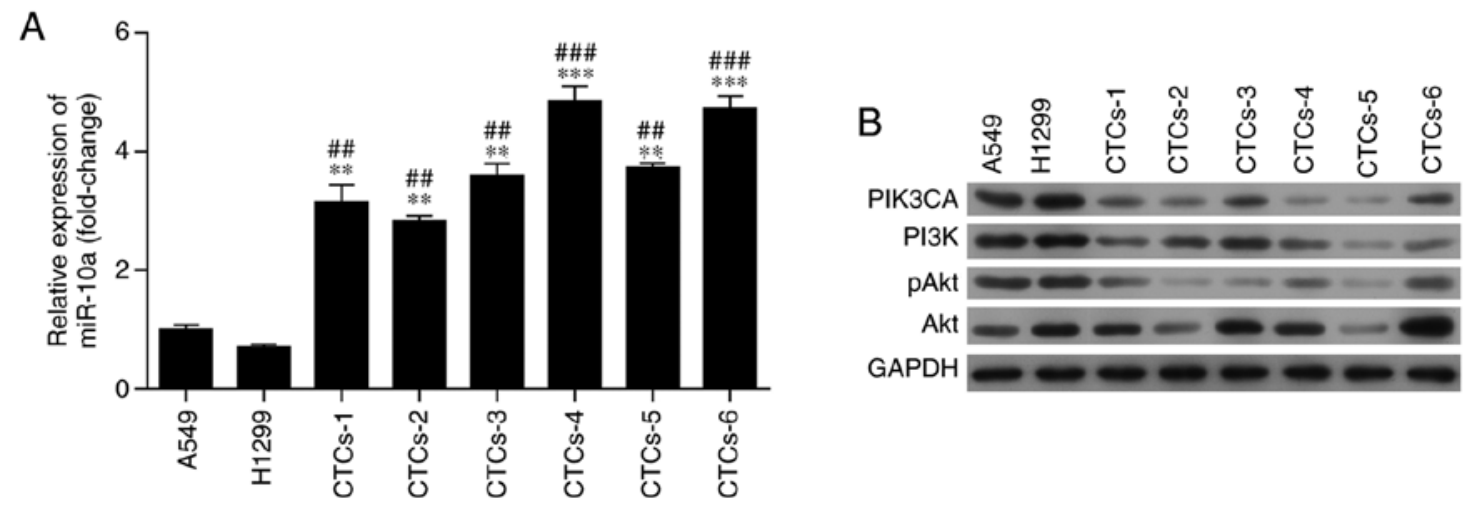

Figure 2. Expression of miR-10a and PIK3CA in lung adenocarcinoma A549 and H1299 cells and CTCs from 6 patients. (A) Expression of miR-10a was determined by RT-qPCR in A549 and A1299 cells and CTCs. (B) Expression of PIK3CA and the activation of the PI3K/Akt signaling pathway were determined by western blotting in A549 and A1299 cells and CTCs. CTCs were isolated from the blood samples obtained from 6 lung adenocarcinoma patients. ${ }^{* *} \mathrm{P}<0.01$ and ${ }^{* * *} \mathrm{P}<0.001$ compared with A549 cells; ${ }^{\# \#} \mathrm{P}<0.01$ and ${ }^{\# \# "} \mathrm{P}<0.001$ compared with H1299 cells. PIK3CA, phosphatidylinositol-4,5-bisphosphate 3-kinase catalytic subunit $\alpha$; PI3K, phosphoinositide 3-kinase; pAkt, phosphorylated protein kinase B; CTCs, circulating tumor cells.
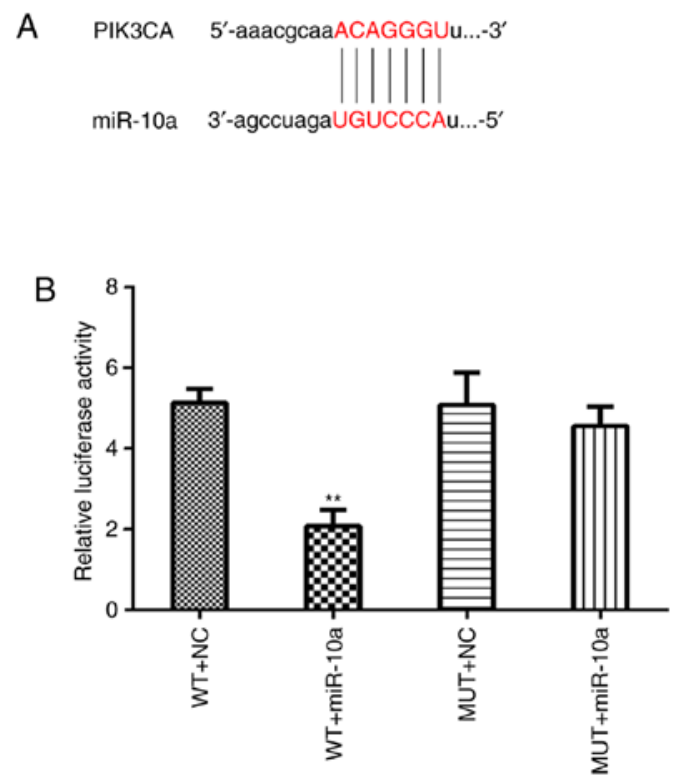

C

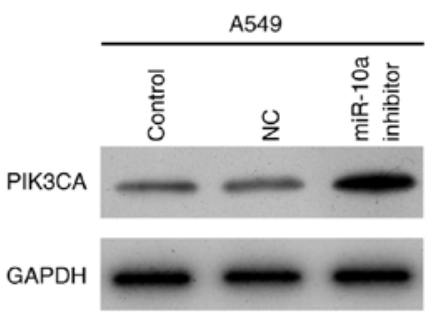

D

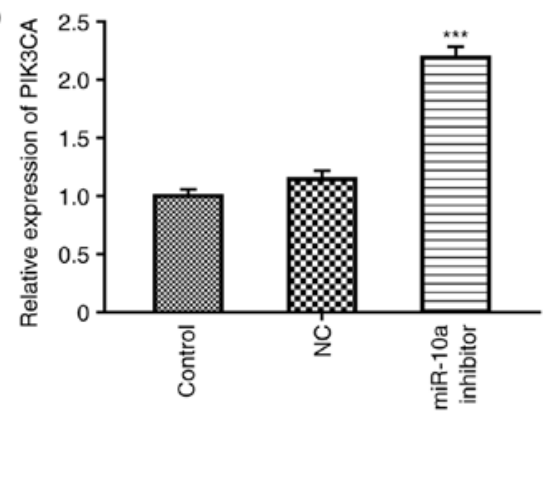

Figure 3. PIK3CA is a target of miR-10a. (A) Prediction of the target regulatory relationship between PIK3CA and miR-10a. (B) Regulatory relationship was confirmed by luciferase reporter assay. WT, wild-type, MUT, mutant; NC, negative control; miR-10a, miR-10a mimic. ${ }^{* *} \mathrm{P}<0.01$ compared with the NC group. (C and D) A549 cells were transfected with the miR-10a inhibitor, and expression of PIK3CA was detected by western blotting and real-time PCR, respectively. GAPDH was used as an internal control. ${ }^{* * *} \mathrm{P}<0.001$ compared with the NC group. PIK3CA, phosphatidylinositol-4,5-bisphosphate 3-kinase catalytic subunit $\alpha$. 

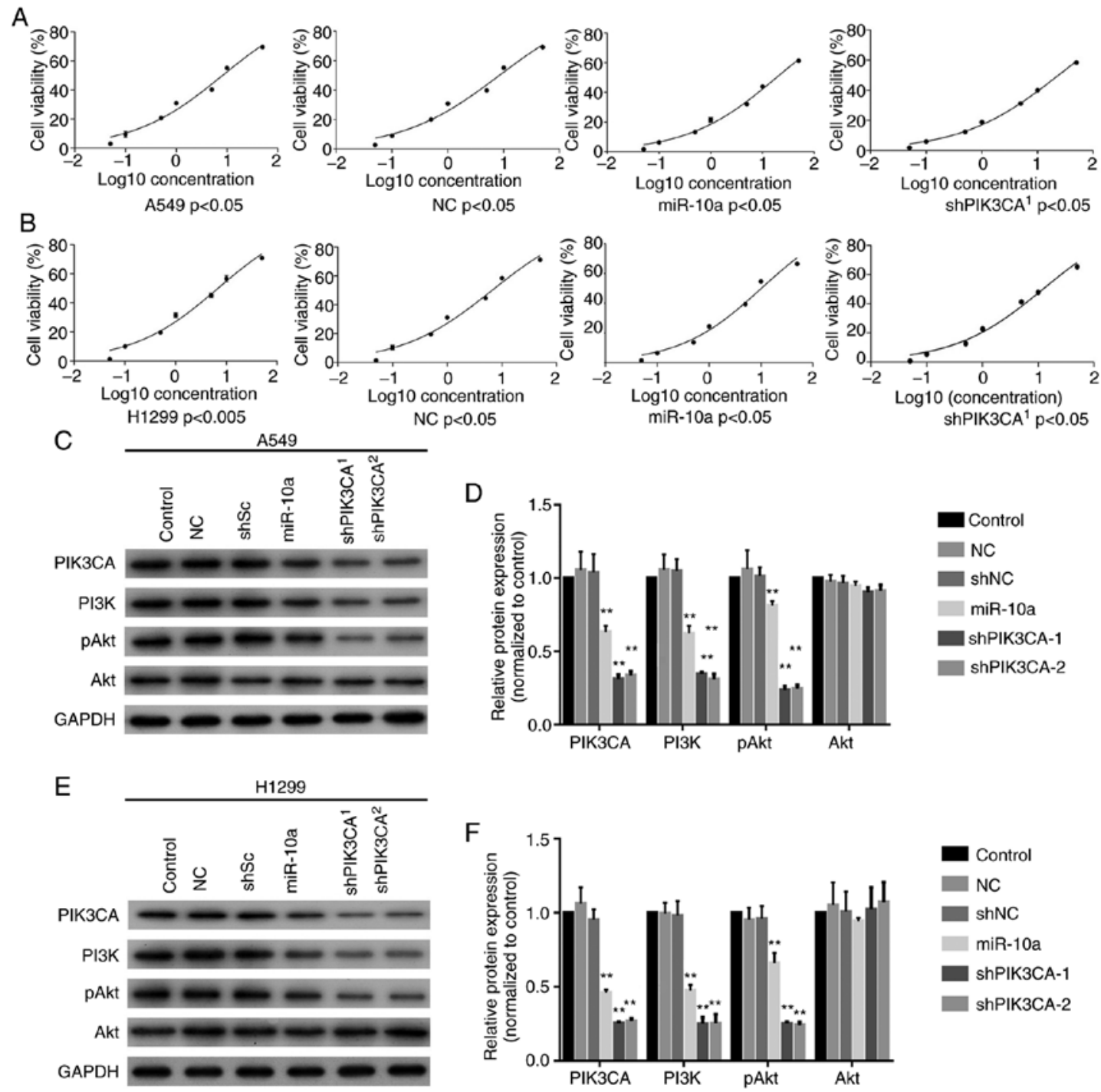

Figure 4. Overexpression of miR-10a enhances the cisplatin resistance of lung adenocarcinoma A549 and H1299 cells. (A) Cisplatin-mediated inhibitory rate of A549 cells after miR-10a overexpression or PIK3CA knockdown (shPIK3CA). (B) Cisplatin-mediated inhibitory rate of H1299 after miR-10a overexpression or PIK3CA knockdown. Expression of PIK3CA and the activation of the PI3K/Akt signaling pathway were determined by western blotting in (C and D) A549 and (E and F) H1299 cells after transfection with miR-10a mimics or shPIK3CA. ${ }^{* *} \mathrm{P}<0.01$ compared with the NC group. PIK3CA, phosphatidylinositol-4,5-bisphosphate 3-kinase catalytic subunit $\alpha$; PI3K, phosphoinositide 3-kinase; pAkt, phosphorylated protein kinase B.

the $\mathrm{A} 549\left(\mathrm{IC}_{50}=8.184\right)$ and $\mathrm{H} 1299$ cells $\left(\mathrm{IC}_{50}=6.80\right)(\mathrm{P}<0.05)$ (Fig. 1). These findings indicated that CTCs may have a lower sensitivity to cisplatin than that of carcinoma cells in lung cancer tissues.

Expression of miR-10a and PIK3CA/PI3K/Akt signaling is altered after treatment with cisplatin. Furthermore, to reveal the underlying mechanism underlying the cisplatin resistance of CTCs, the expression of miR-10a and phosphatidylinositol-4,5-bisphosphate 3-kinase catalytic subunit $\alpha$ (PIK3CA) were detected in CTCs, A549 and H1299 cells after treatment with cisplatin. RT-qPCR revealed that the expression of miR-10a in CTCs was significantly higher than that in A549 and H1299 cells after treatment with cisplatin ( $\mathrm{P}<0.01$, Fig. 2A). Moreover, as determined by western blot analysis, the CTCs exhibited a lower expression of PIK3CA and phosphoinositide 3-kinase (PI3K) than these levels in the A549 and H1299 cells after cisplatin treatment (Figs. 2B and S1). Meanwhile, protein kinase B phosphorylation (pAkt) showed similar results as PIK3CA and PI3K, but no obvious difference was observed in Akt expression (Fig. 2B). These findings suggest that modulation of miR-10a expression and PIK3CA in the PI3K/Akt signaling pathway increases the resistance of CTCs to cisplatin.

PIK3CA is a target of miR-10a. According to miRWalk database, PIK3CA was predicted to be a potential target of miR-10a (http://www.targetscan.org/cgibin/targetscan/vert_71/view_gene. cgi?rs=ENST000 00263967.3\&taxid $=9606 \&$ showcnc $=0 \&$ sh ownc $=0$ \&shownc_nc $=\&$ showncf $1=\&$ showncf $2=\&$ subset $=1 \#$ miR-10-5p, Fig. 3A). Then, the regulatory relationship between miR-10a and PIK3CA was explored using luciferase reporter assay in A549 cells. The results showed that the overexpression of miR-10a significantly decreased the activity of WT-PIK3CA-3'UTR (WT+miR-10a), but not the MUT-PIK3CA-3'UTR (MUT+miR-10a) (Fig. 3B), indicating that PIK3CA is a direct target gene of miR-10a. Finally, A549 cells were transfected with the miR-10a inhibitor, and 

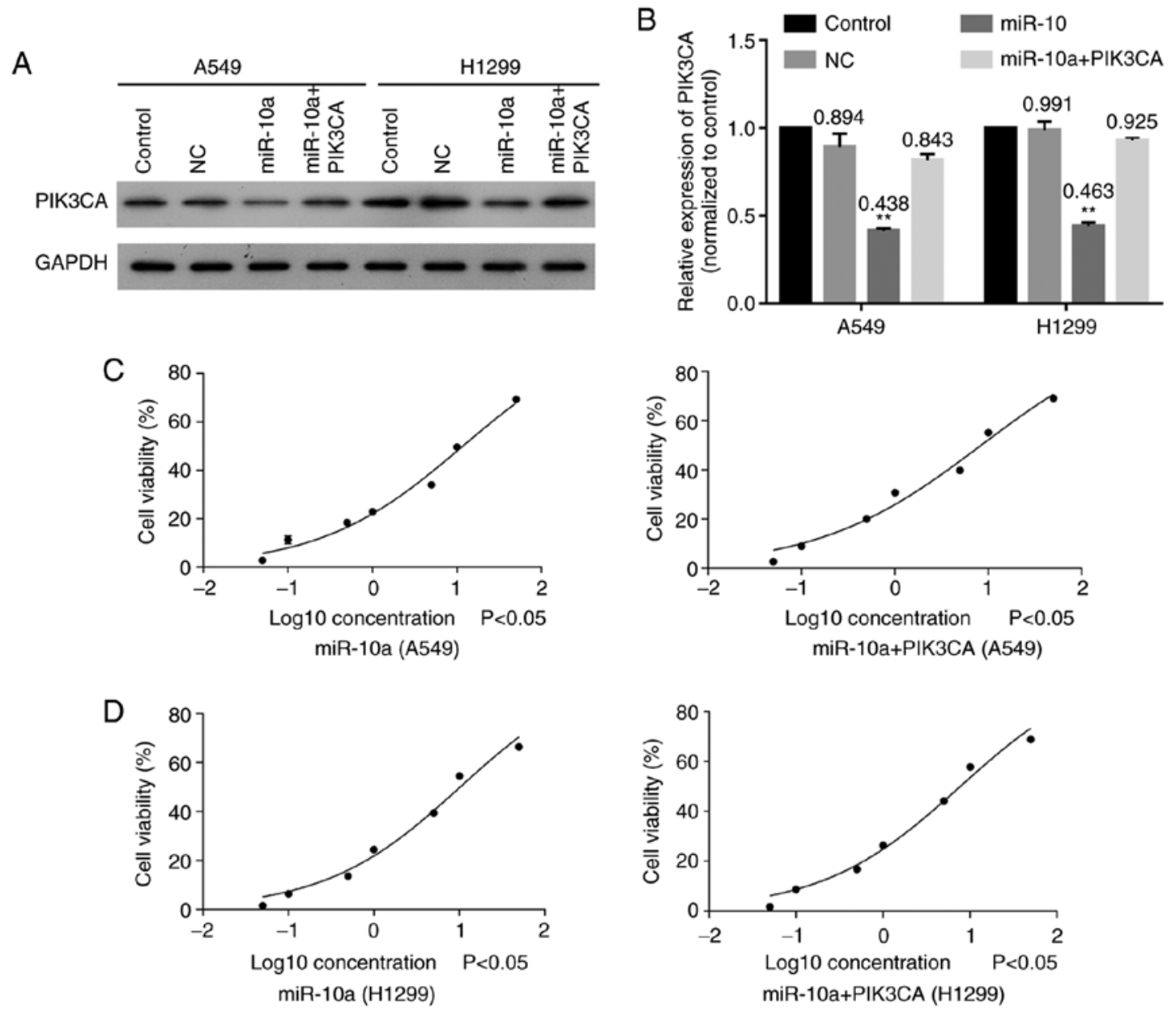

Figure 5. Overexpression of PIK3CA reduces the effect of miR-10a on cisplatin resistance. (A) Expression of PIK3CA in A549 and H1299 cells after overexpression of miR-10a and PIK3CA. (B) Quantitative expression of PIK3CA in A549 and H1299 cells after overexpression of miR-10a and PIK3CA. Cisplatin-mediated inhibitory rate of (C) A549 and (D) H1299 cells after overexpressing miR-10a and PIK3CA. ${ }^{* *}$ P $<0.01$ compared with the NC group. PIK3CA, phosphatidylinositol-4,5-bisphosphate 3-kinase catalytic subunit $\alpha$.

expression of PIK3CA was detected by western blotting and real-time PCR, respectively. The results showed that miR-10a inhibitor increased both the protein and mRNA levels of PIK3CA (Fig. 3C and D).

Overexpression of miR-10a or silencing of PIK3CA decreases the sensitivity to cisplatin. Next, the miR-10a mimic or shPIK3CA was transfected into A549 and H1299 cells and the sensitivity to cisplatin was determined using CCK-8 assay. The results showed that overexpression of miR-10a $\left(\mathrm{IC}_{50}=18.56\right)$ and knockout of PIK3CA (shPIK3CA) $\left(\mathrm{IC}_{50}=23.77\right)$ significantly increased the $\mathrm{IC}_{50}$ value of A549 cells to cisplatin when compared with the blank control $\left(\mathrm{IC}_{50}=8.184\right)$ and $\mathrm{NC}\left(\mathrm{IC}_{50}=8.366\right.$, Fig. 4A). In addition, overexpression of miR-10a $\left(\mathrm{IC}_{50}=10.14\right)$ and silencing of PIK3CA (shPIK3CA) $\left(\mathrm{IC}_{50}=12.20\right)$ significantly increased the $\mathrm{IC}_{50}$ value of $\mathrm{H} 1299$ to cisplatin compared with the blank control $\left(\mathrm{IC}_{50}=6.86\right)$ and $\mathrm{NC}\left(\mathrm{IC}_{50}=6.56\right.$, Fig. 4B). Moreover, western blotting showed that both miR-10a mimic and shPIK3CA significantly reduced PIK3CA, PI3K and pAkt levels in both the A549 and H1299 cell lines but not the Akt expression level (Fig. 4C-4F). These findings revealed that miR-10a increased the resistance abilities of A549 and H1299 cells to cisplatin by decreasing the expression of PIK3CA in the PI3K/Akt signaling pathway.
Overexpression of PIK3CA increases the sensitivities of A549 and H1299 to cisplatin. Moreover, PIK3CA was also co-transfected with miR-10a mimic into A549 and H1299 cells to explore the effect of the miR-10a/PIK3CA regulatory relationship on sensitivity of lung carcinoma to cisplatin. The results showed that overexpression of miR-10a significantly decreased PIK3CA expression, while overexpression of PIK3CA obviously reversed this effect in both A549 and H1299 cells (Fig. 5A and B). In addition, overexpression of PIK3CA significantly increased the sensitivities of A549 ( $\mathrm{IC}_{50}: 11.69$ vs. 18.56) (Fig. 5C) and H1299 ( $\mathrm{IC}_{50}$ : 7.71 vs. 10.14) (Fig. 5D) cells to cisplatin after transfecting with miR-10 mimic. These findings indicated that overexpression of PIK3CA increased the sensitivities of A549 and H1299 cells to cisplatin.

\section{Discussion}

Recurrence and metastasis of lung cancer are considered as factors of poor prognosis and cancer-related mortality. The presence of circulating tumor cells (CTCs) increases the risk of recurrence and metastasis in patients with lung cancer. Therefore, we isolated CTCs from lung adenocarcinoma blood samples and treated them with cisplatin to detect the resistance of CTCs to cisplatin. Consistent with the increased risk of lung 
cancer recurrence induced by CTCs, we also revealed that the CTCs presented a lower sensitivity to cisplatin than A549 and H1299 cells. These results indicated that the cisplatin resistance of CTCs further lead to the poor prognosis of lung cancer patients.

miRNAs have been widely acknowledged as important regulators of human cancer by serving as oncogenes or tumor suppressors (30). miR-10a is a member of the miR-10a family, and has been found to be involved in multiple malignant transformations, including those of breast cancer (31), glioma (32), liver cancer (33) and pancreatic cancer (34). For instance, Bryant et al revealed that miR-10a is abnormally overexpressed in nucleophomin 1-mutated acute myeloid leukemia (AML) and is positively correlated with AML cell survival (35). Importantly, $\mathrm{Yu}$ et al also revealed that miR-10a is elevated in non-small cell lung cancer (NSCLC) and is positively correlated with the progression of NSCLC via targeting PTEN (36). In addition, Sun et al demonstrated that downregulation of miR-10a expression decreased cisplatin resistance in A549 cells via the TGF- $\beta /$ smad2/STAT3/STAT5 signaling pathway (27). In the present study, miR-10a expression was found to be highly expressed in CTCs when compared to that in A549 and H1299 cells, meaning that the upregulation of miR-10a expression in CTCs may enhance cisplatin resistance of CTCs leading to poor patient prognosis.

Phosphatidylinositol-4,5-bisphosphate 3-kinase catalytic subunit $\alpha$ (PIK3CA) is a p110 $\alpha$ subunit of PI3K and often plays a critical role in regulating the activation of the PI3K/Akt/mTOR signaling pathway. The PI3K/Akt/mTOR pathway is closely related with tumorigenesis in breast cancer and hepatocellular carcinoma by regulating proliferation, migration, metastasis and apoptosis of tumor cells (37-39). Dysregulated PIK3CA expression was found to result in the abnormal activation of the PI3K/Akt pathway and finally in the development of cancer. Herein, we confirmed that PIK3CA expression in CTCs was lower than that in A549 and H1299 cells after treatment with cisplatin, indicating that the malignant biological behavior of CTCs was more significant than that of lung adenocarcinoma cell lines. Hu et al documented that miR-10a could modulate the proliferation of airway smooth muscle cells via directly targeting PIK3CA in the PI3K pathway (40). Moreover, miR-10a was found to suppress the progression of breast cancer via targeting the PIK3CA in the PI3K/Akt/mTOR signaling pathway (41). In addition, PIK3CA was confirmed as a direct target of miR-10a and further revealed that miR-10a overexpression obviously decreased the expression of PIK3CA and PI3K and the phosphorylation of Akt. Moreover, upregulation of PIK3CA largely reversed the effect of miR-10a on the proliferation of A549 and H1299 cells by reducing the expression of PI3K and phosphorylation of Akt. Taken together, this evidence suggests that miR-10a directly regulates the PI3K/Akt signaling pathway via modulating the expression of PIK3CA.

In addition to the regulation of the occurrence and development of multiple cancers, the PI3K/Akt signaling pathway is also closely related to cisplatin resistance, whereby this process includes the participation of many miRNAs. miR-221 has been reported to induce cell survival and cisplatin resistance to human osteosarcoma via the PI3K/Akt signaling pathway (42).
Downregulation of miR-497 was found to contribute to the cell growth and cisplatin resistance via the PI3K/Akt signaling pathway (43). Moreover, Klotho was found to increase lung cancer sensitivity to cisplatin through the PI3K/Akt signaling pathway (44). In the present study, PI3K and phosphorylation of Akt was obviously downregulated, and miR-10a was highly expressed in CTCs when compared with A549 and H1299 cells, indicating that CTCs present a lower sensitivity to cisplatin than A549 and H1299 cells through downregulation of the PI3K/Akt pathway under cisplatin treatment.

There were some limitations to the present study. First, due to the limited sample size, there may have been some bias between this study and the actual situation. Second, although we confirmed that the miR-10a/PIK3CA axis is involved in the pathogenesis of cisplatin resistance of lung cancer, the exact upstream of miR-10a remains unclear. Despite these limitations, our findings provide new information regarding the mechanism of lung cancer resistance to cisplatin.

In conclusion, miR-10a was significantly upregulated in CTCs, exhibiting higher cisplatin resistance than A549 and H1299 cells. Overexpression of miR-10a enhanced the cisplatin resistance of A549 and H1299 and overexpression of PIK3CA largely reversed this effect via the PI3K/Akt signaling pathway. These findings suggest that miR-10a promotes the cisplatin resistance of CTCs by downregulating PIK3CA expression in the PI3K/Akt signaling pathway.

\section{Acknowledgements}

Not applicable.

\section{Funding}

This study was supported by the Shenzhen Healthcare Research Project (no. 201501011) and Guangdong Medical Research Foundation (no. A2016475).

\section{Availability of data and materials}

The datasets used during the present study are available from the corresponding author upon reasonable request.

\section{Authors' contributions}

Conception and design of the study were carried out by $\mathrm{TH}$ and KR. Administrative and experimental support were carried out by TH and GD. Provision of study materials and recruitment of patients and samples were achieved by KR and LY. Collection and assembly of data were carried out by LY and YW. Data analysis and interpretation were performed by BP, GW and ZW. Writing of the manuscript was conducted by all of the authors. All authors read and approved the manuscript and agree to be accountable for all aspects of the research in ensuring that the accuracy or integrity of any part of the work are appropriately investigated and resolved.

\section{Ethics approval and consent to participate}

All patients signed informed consent forms, and this study was authorized by the Ethics Committee of Shenzhen 
People's Hospital, The Second Clinical Medical College of Jinan University (Shenzhen, Guangdong, China). This study was conducted in accordance with the Declaration of Helsinki.

\section{Patient consent for publication}

Not applicable.

\section{Competing interests}

The authors state that they have no competing interests.

\section{References}

1. Siegel RL Miller KD and Jemal A: Cancer Statistics, 2017. CA Cancer J Clin 67: 7-30, 2017.

2. Sorber L, Zwaenepoel K, Deschoolmeester V, Van Schil PE, Van Meerbeeck J, Lardon F, Rolfo C and Pauwels P: Circulating cell-free nucleic acids and platelets as a liquid biopsy in the provision of personalized therapy for lung cancer patients. Lung Cancer 107: 100-107, 2017

3. Gan Y,Zhou P and Li W: Correlations of Tumor Stage and Serum Tumor Markers with Age in Nonsmoking Females with Lung Adenocarcinoma. In: D62 types, Genotypes, and Phenotypes: The Three 'T's' of thoracic oncology. American Thoracic Society, ppA7334, 2018.

4. Zhai X, Zheng Q, Yang L, Zhu Y, Li J, Liu Y and Wang Y: Impact of platinum/pemetrexed combination versus other platinum-based regimens on adjuvant chemotherapy in resected lung adenocarcinoma. Sci Rep 7: 1453, 2017.

5. Jin B, Niu Y, Zhang Y, Chu T, Gu A, Wu J, Pei J, Zhu L and Han B: 1281Pcombination of chemotherapy and gefitinib as first-line treatment of patients with advanced lung adenocarcinoma and sensitive egfr mutations: A randomised controlled trial. Ann Oncol 25 (4 Suppl): iv451, 2014.

6. Xu R, Mao Y, Chen K, He W, Shi W and Han Y: The long noncoding RNA ANRIL acts as an oncogene and contributes to paclitaxel resistance of lung adenocarcinoma A549 cells. Oncotarget 8: 39177-39184, 2017.

7. Sato C, Okuda K, Tamiya H, Yamamoto K, Hoshina K, Narumoto O, Urushiyama H, Noguchi S, Amano Y, Watanabe $\mathrm{K}$, et al: Acute arterial thrombosis during postoperative adjuvant cisplatin-based chemotherapy for completely resected lung adenocarcinoma. Intern Med 57: 557-561, 2018.

8. Wang Q, Cheng N, Li X, Pan H, Li C, Ren S, Su C, Cai W, Zhao C, Zhang L and Zhou C: Correlation of long non-coding RNA H19 expression with cisplatin-resistance and clinical outcome in lung adenocarcinoma. Oncotarget 8: 2558-2567, 2017.

9. Raz DJ, Zell JA, Ou SH, Gandara DR, Anton-Culver H and Jablons DM: Natural history of stage Inon-small cell lung cancer:Implications for early detection. Chest 132: 193-199, 2007.

10. Roselli M, Mariotti S, Ferroni P, Laudisi A, Mineo D, Pompeo E, Ambrogi V and Mineo TC: Postsurgical chemotherapy in stage IB non-small cell lung cancer: Long-term survival in a randomized study. Int J Cancer 119: 955-960, 2006.

11. García SA, Weitz J and Schölch S: Circulating tumor cells. In: Cancer stem cells: Methods and protocols. Papaccio G and Desiderio V (eds). Springer, New York, NY, pp213-219, 2018.

12. Yu M, Bardia A, Wittner BS, Stott SL, Smas ME, Ting DT, Isakoff SJ, Ciciliano JC, Wells MN, Shah AM, et al: Circulating breast tumor cells exhibit dynamic changes in epithelial and mesenchymal composition. Science 339: 580-584, 2013.

13. Kallergi G, Papadaki MA, Politaki E, Mavroudis D, Georgoulias V and Agelaki S: Epithelial to mesenchymal transition markers expressed in circulating tumour cells of early and metastatic breast cancer patients. Breast Cancer Res 13: R59, 2011.

14. Giuliano M, Giordano A, Jackson S, Hess KR, De Giorgi U, Mego M, Handy BC, Ueno NT, Alvarez RH and De Laurentiis M: Circulating tumor cells as prognostic and predictive markers in metastatic breast cancer patients receiving first-line systemic treatment. Breast Cancer Res 13: R67, 2011.
15. Pierga JY, Hajage D, Bachelot $T$, Delaloge $S$, Brain $E$, Campone M, Diéras V, Rolland E, Mignot L, Mathiot C and Bidard FC: High independent prognostic and predictive value of circulating tumor cells compared with serum tumor markers in a large prospective trial in first-line chemotherapy for metastatic breast cancer patients. Ann Oncol 23: 618-624, 2012.

16. Yokobori T, Iinuma H, Shimamura T, Imoto S, Sugimachi K, Ishii H, Iwatsuki M, Ota D, Ohkuma M, Iwaya T, et al: Plastin3 is a novel marker for circulating tumor cells undergoing the epithelial-mesenchymal transition and is associated with colorectal cancer prognosis. Cancer Res 73: 2059-2069, 2013.

17. Lianidou ES, Markou A and Strati A: The role of CTCs as tumor biomarkers. Adv Exp Med Biol 867: 341-367, 2015.

18. Xia H, Ooi LL and Hui KM: MicroRNA-216a/217-induced epithelial-mesenchymal transition targets PTEN and SMAD7 to promote drug resistance and recurrence of liver cancer. Hepatology 58: 629-641, 2013.

19. Zhu W, Zhu D, Lu S, Wang T, Wang J, Jiang B, Shu Y and Liu P: miR-497 modulates multidrug resistance of human cancer cell lines by targeting BCL2. Med Oncol 29: 384-391, 2012.

20. Zhu W, Xu H, Zhu D, Zhi H, Wang T, Wang J, Jiang B, Shu Y and Liu P: miR-200bc/429 cluster modulates multidrug resistance of human cancer cell lines by targeting BCL2 and XIAP. Cancer Chemother Pharmacol 69: 723-731, 2012.

21. Chen QY JD, Wang J, Hu H, Tang X, Chen J, Mou H and Lu W: miR-206 regulates cisplatin resistance and EMT in human lung adenocarcinoma cells partly by targeting MET. Oncotarget 7: 24510-24526, 2016.

22. Zhao Z, Zhang L, Yao Q and Tao Z: miR-15b regulates cisplatin resistance and metastasis by targeting PEBP4 in human lung adenocarcinoma cells. Cancer Gene Ther 22: 108-114, 2015.

23. Kitamura K, Seike M,OkanoT,Matsuda K, Miyanaga A,MizutaniH, Noro R, Minegishi Y, Kubota K and Gemma A: MiR-134/487b/655 cluster regulates TGF- $\beta$-induced epithelial-mesenchymal transition and drug resistance to gefitinib by targeting MAGI 2 in lung adenocarcinoma cells. Mol Cancer Ther 13: 444-453, 2014.

24. Ujifuku K, Mitsutake N, Takakura S, Matsuse M, Saenko V, Suzuki K, Hayashi K, Matsuo T, Kamada K, Nagata I and Yamashita S: miR-195, miR-455-3p and miR-10a(*) are implicated in acquired temozolomide resistance in glioblastoma multiforme cells. Cancer Lett 296: 241-248, 2010.

25. Bao M, Pan S, Yang W, Chen S, Shan Y and Shi H: Serum mir-10a-5p and mir-196a-5p as non-invasive biomarkers in non-small cell lung cancer. Int J Clin Exp Pathol 11: 773-780, 2018.

26. Liu Y, Xu N, Liu B, Huang Y, Zeng H, Yang Z, He Z and Guo H: Long noncoding RNA RP11-838N2.4 enhances the cytotoxic effects of temozolomide by inhibiting the functions of miR-10a in glioblastoma cell lines. Oncotarget 7: 43835-43851, 2016.

27. Sun W, Ma Y, Chen P and Wang D: MicroRNA-10a silencing reverses cisplatin resistance in the A549/cisplatin human lung cancer cell line via the transforming growth factor- $\beta /$ Smad2/STAT3/STAT5 pathway. Mol Med Rep 11: 3854-3859, 2015.

28. Gong C, Liu B, Yao Y, Qu S, Luo W, Tan W, Liu Q, Yao H, Zou L, Su F and Song E: Potentiated DNA damage response in circulating breast tumor cells confers resistance to chemotherapy. J Biol Chem 290: 14811-14825, 2015.

29. Livak KJ and Schmittgen TD: Analysis of relative gene expression data using real-time quantitative PCR and the 2(-Delta Delta C(T)) methods. Methods 25: 402-408, 2001.

30. Damavandi Z, Torkashvand S, Vasei M, Soltani BM, Tavallaei M and Mowla SJ: Aberrant expression of breast development-related MicroRNAs, miR-22, miR-132, and miR-212, in breast tumor tissues. J Breast Cancer 19: 148-155, 2016.

31. Ma L, Teruya-Feldstein J and Weinberg RA: Tumour invasion and metastasis initiated by microRNA-10b in breast cancer. Nature 449: 682-688, 2007.

32. Yan Y, Wang Q, Yan XL, Zhang Y, Li W, Tang F, Li X and Yang P: miR-10a controls glioma migration and invasion through regulating epithelial-mesenchymal transition via EphA8. FEBS Lett 589: 756-765, 2015.

33. Wang Y, Liu Z, Yao B, Dou C, Xu M, Xue Y, Ding L, Jia Y, Zhang $\mathrm{H}$ and Li Q: Long non-coding RNA TUSC7 acts a molecular sponge for miR-10a and suppresses EMT in hepatocellular carcinoma. Tumour Biol 37: 11429-11441, 2016.

34. Weiss FU, Marques IJ, Woltering JM, Vlecken DH, Aghdassi A, Partecke LI, Heidecke CD, Lerch MM and Bagowski CP: Retinoic acid receptor antagonists inhibit miR-10a expression and block metastatic behavior of pancreatic cancer. Gastroenterology 137: 2136-2145.e1-7, 2009. 
35. Bryant A, Palma CA, Jayaswal V, Yang YW, Lutherborrow M and Ma DD: miR-10a is aberrantly overexpressed in Nucleophosmin1 mutated acute myeloid leukaemia and its suppression induces cell death. Mol Cancer 11: 8, 2012.

36. Yu T, Liu L, Li J, Yan M, Lin H, Liu Y, Chu D, Tu H, Gu A and Yao M: MiRNA-10a is upregulated in NSCLC and may promote cancer by targeting PTEN. Oncotarget 6: 30239-30250, 2015.

37. Janku F, Wheler JJ, Westin SN, Moulder SL, Naing A, Tsimberidou AM, Fu S, Falchook GS, Hong DS, Garrido-Laguna I, et al: PI3K/AKT/mTOR inhibitors in patients with breast and gynecologic malignancies harboring PIK3CA mutations. J Clin Oncol 30: 777-782, 2012.

38. Woo SU, Sangai T, Akcakanat A, Chen $\mathrm{H}$, Wei $\mathrm{C}$ and Meric-Bernstam F: Vertical inhibition of the PI3K/Akt/mTOR pathway is synergistic in breast cancer. Oncogenesis 6: e385, 2017.

39. Chai R, Fu H, Zheng Z, Liu T, Ji S and Li G: Resveratrol inhibits proliferation and migration through SIRT1 mediated post-translational modification of PI3K/AKT signaling in hepatocellular carcinoma cells. Mol Med Rep 16: 8037-8044, 2017.
40. Hu R, Pan W, Fedulov AV, Jester W, Jones MR, Weiss ST, Panettieri RA Jr, Tantisira K and Lu Q: MicroRNA-10a controls airway smooth muscle cell proliferation via direct targeting of the PI3 kinase pathway. FASEB J 28: 2347-2357, 2014.

41. Ke K and Lou T: MicroRNA-10a suppresses breast cancer progression via PI3K/Akt/mTOR pathway. Oncol Lett 14: 5994-6000, 2017.

42. Zhao G, Cai C, Yang T, Qiu X, Liao B, Li W, Ji Z, Zhao J, Zhao H, Guo M, et al: MicroRNA-221 induces cell survival and cisplatin resistance through PI3K/Akt pathway in human osteosarcoma. PLoS One 8: e53906, 2013.

43. Shao XJ, Miao MH, Xue J, Xue J, Ji XQ and Zhu H: The Down-regulation of MicroRNA-497 contributes to cell growth and cisplatin resistance through PI3K/Akt pathway in osteosarcoma. Cell Physiol Biochem 36: 2051-2062, 2015.

44. Wang Y, Chen L, Huang G, He D, He J, Xu W, Zou C, Zong F, Li Y, Chen B, et al: Klotho sensitizes human lung cancer cell line to cisplatin via PI3k/Akt pathway. PLoS One 8: e57391, 2013.

(i) $\Theta$ This work is licensed under a Creative Commons Attribution-NonCommercial-NoDerivatives 4.0 International (CC BY-NC-ND 4.0) License. 